\title{
Muscle Latency and Proprioception in Non-Dominant and Dominant Legs of Healthy Sedentary Individuals
}

\author{
Ufuk ŞEKIR1, Saadet Banu KELEŞ², Hakan GÜR \\ 'Department of Sports Medicine, Uludağ University Faculty of Medicine, Bursa, Turkey \\ ${ }^{2}$ Department of Sports Medicine, Bursa Şevket YIlmaz Training and Research Hospital, Bursa, Turkey
}

\begin{abstract}
Objective: The effects of lower extremity dominance on response latency and proprioceptive ability have been reported for physically active individuals, but not for sedentary individuals. The aim of this research was to explore the differences in muscle latency and proprioceptive ability between the non-dominant and dominant legs of healthy sedentary individuals.

Material and Methods: Nineteen healthy male subjects without a history of any physical training practice for a minimum of 12 months were enrolled in this study. An ankle inversion tilting platform was used to measure the reaction times of the tibialis anterior and peroneus longus muscles. Joint position sense measured actively and passively and kinesthesia were used to evaluate proprioception of the ankle joint.

Results: Neither the latency times of the tibialis anterior and peroneus longus muscles nor the proprioceptive ability score measurements exhibited significant differences between the non-dominant and dominant legs $(p>0.05)$.

Conclusion: The results of this investigation indicates that there is no side differences between the limbs with the evaluation of the peroneus longus or tibialis anterior muscle reaction times, ankle joint position sense, and ankle kinesthesia for healthy sedentary individuals.

Keywords: Muscle reaction time, joint position sense, kinesthesia, dominance
\end{abstract}

\section{Introduction}

Impaired proprioception (1-3), postural control (4-6), muscle strength $(7,8)$, and prolonged peroneal reaction time (9-11) of the ankle are reported to be the primary causes of functional ankle instability and ankle sprains. Therefore, evaluating these risk factors in healthy individuals is important to protect and rehabilitate the ankle. When healthy subjects are in question, the clinicians mostly prefer to evaluate or measure the strength, proprioceptive ability, or muscle latency of the dominant ankle (12-14). Recently, however, it was shown that the latency of the peroneus longus muscle was significantly different between the non-dominant and dominant ankles in healthy physically active individuals, with the dominant ankle having a longer latency than the non-dominant ankle (15). Similarly, Knight et al. (16) also reported that the nondominant limb has improved proprioceptive ability and balance over the dominant limb in physically active individuals. On the other hand, Lin et al. (17) did not find any differences in the other risk factors for ankle sprains, such as ankle strength and balance, between the non-dominant and dominant ankles in healthy adults who did not perform any regular physical training practice. These different results between the non-dominant and dominant ankles of healthy individuals suggest that athletes and physically active individuals place different demands on one limb compared with that on the other. 
If the possible differences in sensorimotor control between the limbs are due to activity related demands or improvements in athletes needs to be clarified. On the contrary to some studies showing differences in muscle latency and balance between the limbs in physically active individuals $(15,16)$, comparisons between the limbs in terms of ankle muscle strength or balance represented no differences in sedentary individuals (1719). Similarly, because of the anticipation that similar demands would be placed on the non-dominant and dominant ankles in sedentary individuals, we hypothesized that muscle latency and proprioceptive ability also would not be different between the limbs in sedentary individuals.

Therefore, the aim of this investigation was to explore the differences in muscle latency and proprioceptive ability between the non-dominant and dominant ankles of healthy sedentary individuals. It is important to know the magnitude of the differences between the non-dominant limb and dominant limb in inactive individuals. When this is known, a comparison between an injured ankle and its contralateral healthy ankle and the development of protective approaches, whether it is the dominant or non-dominant limb, can be performed with more accuracy.

\section{Material and Methods}

\section{Participants}

Nineteen healthy male individuals were enrolled to this study (mean age, 23.2 \pm 3.1 years; mean height, $176.1 \pm 4.7 \mathrm{~cm}$; mean body weight, $72.7 \pm 9.0 \mathrm{~kg}$ ). After being informed of the study and test procedures and any possible risks and discomfort that may ensue, all of the participants read and signed an informed consent form that had been approved by the Ethical Board for Protection of Human Subjects of Uludağ University Faculty of Medicine, which also approved the study. None of the participants that were included to this study performed any type of physical training for a minimum of 12 months prior to this study. Thus, it was possible to eliminate incidental training effects that may confound the test results. Participants were included in the study if they met the following criteria: did not have or experience an ankle sprain, low back or lower extremity dysfunction, any ankle surgery or fracture or any occurrence of "giving way", complained of pain, swelling, or functional limitations in the ankles, or took part in any therapeutic exercise for the ankles within the preceding 12 months. All of the participants had no mechanical ankle instability prior to participating in the study according to anterior drawer and talar tilt tests performed by the same clinician. To be consistent with previous research studies, the leg that the participant uses to naturally kick a ball was defined as the dominant leg $(11,15,18,20-23)$. The order of testing (non-dominant or dominant limb first) was randomly determined.

\section{Experimental Procedure}

All the tests were performed on two separate days. Reaction time of the peroneus longus and the tibialis anterior muscles were measured on the first day. Thereafter, evaluation of the proprioceptive ability of the ankle joint was performed on the second day.

\section{Muscle Reaction Time Measurements}

A detailed description of the same muscle reaction time measurement protocol used in this study, including instrumentation, participant preparation, and testing procedure, has been published previously by Keles et al. (24). Briefly, reaction time of the peroneus longus and tibialis anterior muscles were measured using surface EMG during sudden ankle supination that was provided with a custom-built trapdoor mechanism. The following different supinating conditions that were chosen to evaluate muscle reaction time were $15^{\circ}$ inversion during ankle in neutral (0015) and $20^{\circ}$ of plantarflexion (2015) and $30^{\circ}$ inversion during ankle in neutral (0030) and $20^{\circ}$ of plantarflexion (2030) (Figure 1a, b). Muscle onset or reaction time was measured between the latency of the moment of ankle inversion to the first EMG response (Figure 2). The average of three measurements was used for analysis.

\section{Reliability of the Tilting Platform}

The reliability of the tilting platform was previously evaluated in a pilot study performed in our laboratory (25). This study evaluated the reliability of the reaction time measurements of the peroneal muscles from the ankle in neutral position to $30^{\circ}$ inversion. The reliability of the test was calculated using intraclass correlation coefficients (ICCs). Currier accepted the ICC as clinically meaningful if its value is $\geq 0.80$ (26). As a result, good reliability was obtained for the peroneal reaction time according to the ICC (ICC=0.80) (25).

\section{Proprioceptive Ability of the Ankle Joint}

Joint position sense in active and passive mode and kinesthesia were measured to evaluate proprioception of the ankle joint.

\section{Ankle Joint Position Sense}

Joint position sense of the ankle was measured using a computerized isokinetic dynamometer (Cybex NORM ${ }^{\mathrm{TM}}$, CSMI, USA) at a $0.5^{\circ} \cdot \mathrm{s}^{-1}$ angular speed. To evaluate active and passive ankle joint position senses, $10^{\circ}$ and $20^{\circ}$ of ankle inversion and $15^{\circ}$ and $30^{\circ}$ of ankle plantarflexion were selected as the test angles. Details for the subject positioning on the dynamometer and testing procedure are described in the studies of Sekir et al. $(8,27)$. Briefly, the subject was asked to reproduce one of the requested test angles either actively or passively after the tester has passively moved the tested ankle from neutral position to the testing position, held it for $5 \mathrm{~s}$, and moved it back passively from the presented angle to the reference angle (neutral position). Error score in degrees that existed between the reference and repositioned test angle was recorded as angular displacement. The over- or underestimated angular displacements from the reference angle were not considered in calculating the error scores. Thus, only the absolute errors were noted. Each tested condition was repeated three times and the mean was calculated to determine the average absolute error score. 
Table 1. Muscle reaction time in the dominant and non-dominant $\operatorname{limb}[$ Mean \pm SD]

\begin{tabular}{lcccc} 
& Dominant & Non-dominant & F value & p value \\
\hline PerRT0030 (msec) & $97.1 \pm 23.9$ & $85.5 \pm 13.7$ & 3.357 & 0.075 \\
PerRT0015 (msec) & $101.8 \pm 29.8$ & $91.3 \pm 13.1$ & 1.989 & 0.167 \\
PerRT2030 (msec) & $88.4 \pm 9$ & $87.4 \pm 16.3$ & 0.061 & 0.806 \\
PerRT2015 (msec) & $91.3 \pm 22.8$ & $88.7 \pm 18.3$ & 0.153 & 0.698 \\
TibRT0030 (msec) & $101.3 \pm 27.3$ & $90 \pm 18.6$ & 2.253 & 0.144 \\
TibRT0015 (msec) & $104.5 \pm 26$ & $100.5 \pm 18.4$ & 0.291 & 0.593 \\
TibRT2030 (msec) & $94.7 \pm 22.2$ & $92.4 \pm 14.1$ & 0.154 & 0.697 \\
TibRT2015 (msec) & $101.1 \pm 30.4$ & $96.8 \pm 22.1$ & 0.238 & 0.629
\end{tabular}

PerRT: Peroneal reaction time; TibRT: tibial reaction time; 0030: neutral, $30^{\circ}$ inversion; 0015: neutral, $15^{\circ}$ inversion; $2030: 20^{\circ}$ plantarflexion, $30^{\circ}$ inversion; 2015 : $20^{\circ}$ plantarflexion, $15^{\circ}$ inversion

\section{Kinesthesia}

Kinesthesia is evaluated by determining the threshold for detecting passive motion in the joint and was measured using the continuous passive motion (CPM) mode of the isokinetic dynamometer (Cybex NORM ${ }^{\mathrm{TM}}$, CSMI, USA) at an angular speed of $0.1^{\circ} \cdot \mathrm{s}^{-1}$. The same participant positioning on the isokinetic dynamometer's platform used in the measurements of sensitivity to joint position was also used in this test.

The test was started when the foot was positioned in the neutral $\left(0^{\circ}\right)$ position. After the participants were blindfolded and were ready for testing, the dynamometer began to move continuously from the neutral position to inversion or plantarflexion at an angular speed of $0.1^{\circ} \cdot \mathrm{s}^{-1}$ at any time over a period of $1 \mathrm{~min}$. The participants were asked to identify passive motion or a change in joint position. When they felt any motion, they were told to signal the tester. Three trials were performed. The threshold for detecting passive motion was determined as the error in degrees between the starting angle (neutral position) and the angle where the subject detected passive motion. Each tested condition was repeated three times and the mean was calculated to determine the average error score.

\section{Statistical Analysis}

Significant differences in all the variables between the dominant and non-dominant limbs were calculated using one-way analysis of variance (ANOVA). The level of significance was set to $p<0.05$. The statistical analysis was performed using Statistical Package for the Social Sciences version 16.0 (SPSS Inc., Chicago, IL, USA). All of the tests were two-tailed.

\section{Results}

\section{Muscle Reaction Time}

The descriptive statistics for the peroneus longus and tibialis anterior reaction times for the non-dominant and dominant limbs in neutral and plantar-flexed ankle positions are presented in Table 1. Although all the muscle reaction times were slightly lower for the non-dominant limb, no statistically significant differences were observed between the limbs ( $p>0.05)$.
Table 2. Joint position sense and kinesthesia scores in the dominant and non-dominant limb [Mean \pm SD]

\begin{tabular}{lcccc} 
& Dominant & Non-dominant & F value & p value \\
\hline PasJPS20-In (degree) & $2.1 \pm 1.5$ & $2.1 \pm 1.2$ & 0.031 & 0.862 \\
PasJPS10-In (degree) & $1.8 \pm 1.6$ & $1.3 \pm 0.7$ & 1.501 & 0.229 \\
ActJPS20-In (degree) & $1.8 \pm 1.3$ & $2.3 \pm 1.4$ & 1.340 & 0.255 \\
ActJPS10-In (degree) & $1.7 \pm 0.9$ & $1.4 \pm 1$ & 1.361 & 0.251 \\
PasJPS30-PF (degree) & $3.8 \pm 2$ & $3.1 \pm 2$ & 1.406 & 0.243 \\
PasJPS15-PF (degree) & $2 \pm 1.5$ & $2.1 \pm 1.4$ & 0.113 & 0.738 \\
ActJPS30-PF (degree) & $3.6 \pm 2.3$ & $2.7 \pm 1.6$ & 2.309 & 0.137 \\
ActJPS15-PF (degree) & $1.8 \pm 1.1$ & $1.6 \pm 1.1$ & 0.524 & 0.474 \\
TDPM-In (degree) & $0.7 \pm 0.4$ & $0.8 \pm 0.6$ & 0.417 & 0.523 \\
TDPM-PF (degree) & $0.6 \pm 0.3$ & $0.8 \pm 0.5$ & 1.265 & 0.268 \\
\hline
\end{tabular}

ActJPS10-In: Active ankle joint position sense at $10^{\circ}$ of inversion angle ActJPS20-In: Active ankle joint position sense at $20^{\circ}$ of inversion angle PasJPS10-In: Passive ankle joint position sense at $10^{\circ}$ of inversion angle PasJPS20-In: Passive ankle joint position sense at $20^{\circ}$ of inversion angle ActJPS15-PF: Active ankle joint position sense at $15^{\circ}$ of plantarflexion angle ActJPS30-PF: Active ankle joint position sense at $30^{\circ}$ of plantarflexion angle PasJPS15-PF: Passive ankle joint position sense at $15^{\circ}$ of plantarflexion angle PasJPS30-PF: Passive ankle joint position sense at $30^{\circ}$ of plantarflexion angle TDPM: Threshold to detect passive motion
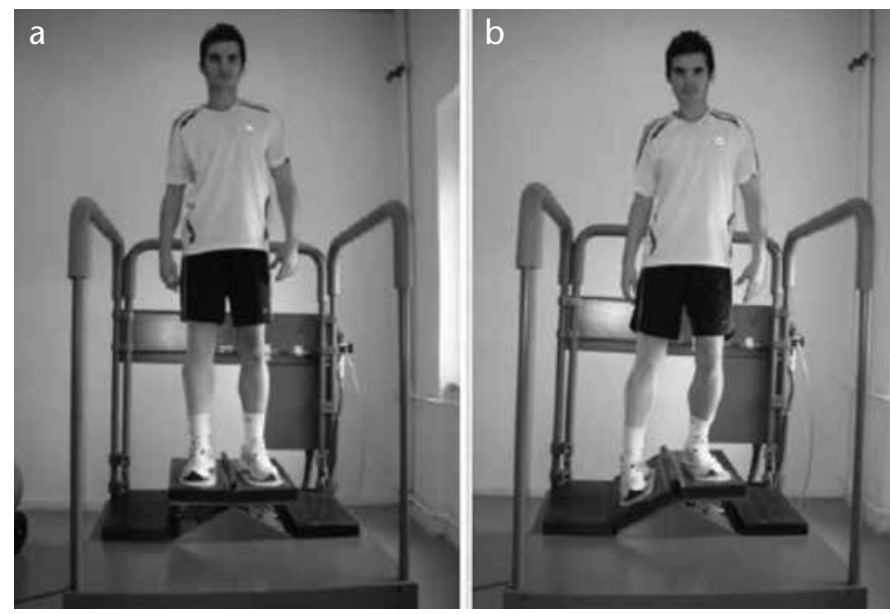

Figure 1. a,b. Subject on the custom-built trap-door mechanism (a) before and (b) after sudden ankle supination of $30^{\circ}$ of inversion from the ankle in neutral position (supination condition 0030)

\section{Proprioceptive Ability}

The active and passive reproduction error scores for the ankle joint position sense for $10^{\circ}$ and $20^{\circ}$ of ankle inversion and $15^{\circ}$ and $30^{\circ}$ of ankle plantarflexion and the scores for the threshold to detect passive motion, either to inversion or plantarflexion, are presented in Table 2. As shown in the table, ANOVA showed no significant differences between the non-dominant and dominant ankles for all the measured proprioceptive ability scores ( $p>0.05)$. 


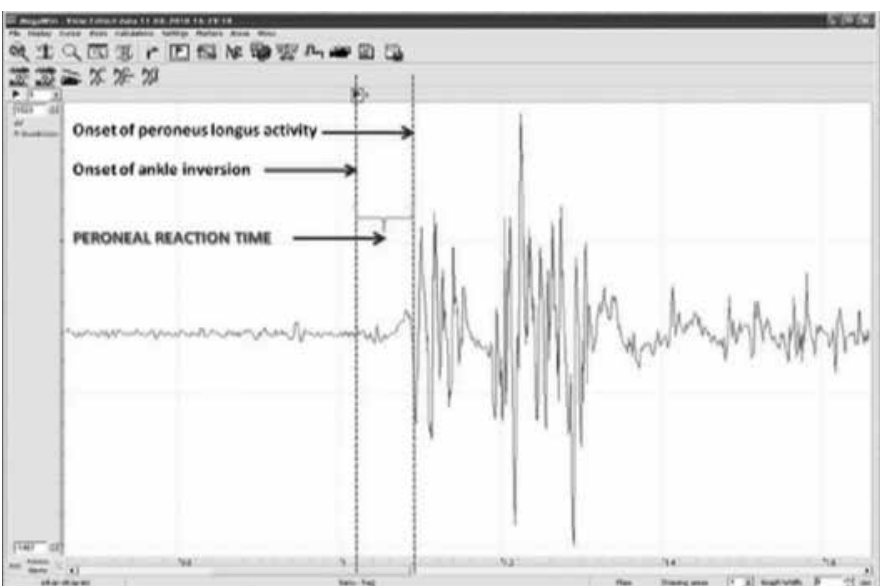

Figure 2. Sample tracing of raw electromyographic activity presents the calculation of peroneal reaction time during sudden ankle supination

\section{Discussion}

The aim of this investigation was to explore whether the muscle latencies and proprioceptive ability of the ankle joint differ between the limbs of sedentary healthy participants. Because it was shown that the different demands placed upon the non-dominant and dominant limbs may cause measurable differences in postural control and response latency in physically active individuals, we hypothesized that muscle latency and proprioceptive ability would not be different between the limbs in sedentary individuals because of the anticipation that similar demands would be placed on both legs in these individuals. In accordance with our hypothesis, the results of this investigation presented no significant differences in the latency times of the peroneus longus and tibialis anterior muscles and the proprioceptive ability of the ankle joint between the non-dominant and dominant ankles of healthy sedentary individuals.

The present study exhibited partially consistent results with the previous studies for reaction times for the peroneal and tibialis anterior muscles $(10,28-31)$. In this study, the mean peroneal latencies ranged from 88 to $102 \mathrm{msec}$ and 86 to $91 \mathrm{msec}$ in the non-dominant and dominant limbs, respectively. Although shorter peroneal reaction times (50-66 msec) in healthy ankles compared to our results were stated in a majority of the previous studies $(10,28,31,32)$, the results of other studies $(29,30)$ were quite comparable with our results. The mean anterior tibial latencies ranged from 95 to $105 \mathrm{msec}$ and 90 to $101 \mathrm{msec}$ in the non-dominant and dominant limbs, respectively. The reaction times in the tibialis anterior muscle were also longer than the results of previous studies (55-80 $\mathrm{msec})$, which were measured in healthy ankles (10,33-35). However, Lynch et al. (29) found similar results (88-107 $\mathrm{msec}$ ) to our study. Different locations in electrode placement, the style of the trapdoor system, different calculations in muscle latency, testing methods while standing or walking, or the body weight distribution on the platform used in the previous studies could be the reasons for the discrepancies. Because it could affect activations of the peroneal and tibial muscles, the expectation of the release of the trap door mechanism by the subject may be another reason. The subjects in this study were asked to turn their back to the tester while on the platform to eliminate this possible influence during testing. Thus, the subjects did not have the chance to see the release of the platform.

Studies investigating the effects of limb dominance in healthy sedentary individuals without any physical training experience mostly focused on ankle strength and postural balance (17-19). These studies have shown no differences between the limbs for the risk factors for ankle sprain, such as ankle strength and postural balance measures. Although muscle latency is not directly linked with strength and balance, our results showing no difference between the limbs in muscle latency correlate with those of previous studies with respect to limb dominance (17-19). Limb dominance was studied by Lin et al. (17). They studied ankle strength and unilateral static balance control in individuals having no attendance in any type of physical training practice. Their results indicate that there are no differences between the nondominant and dominant ankles with respect to invertor and evertor strengths and static balance. Acute or chronic injuries and diseases rather than limb dominance were suggested by the authors for the asymmetries in strength or single-leg balance test in healthy sedentary individuals (17). In another study, Alonso et al. (18) also could not demonstrate any differences in postural balance between the limbs in volunteers who were not involved in any physical activity for a minimum of 6 months before the study. According to the evaluation of single-leg balance, the authors stated that there is no side differences between the limbs in sedentary healthy individuals whose physical activities consists of only daily activities and walking (18). Similarly, Hoffman et al. (19) aimed to evaluate postural control with a singlelimb stance for non-dominant and dominant ankles in healthy inactive individuals. Their results also showed no differences between the non-dominant and dominant legs.

With respect to latency measures, there is currently a lack of knowledge on the effects of leg dominance on muscle reaction times of the peroneus longus or tibialis anterior muscles in healthy and sedentary individuals. One study in the literature concentrated on the difference in the latency of the peroneus longus muscle between the non-dominant and dominant limbs in physically active individuals participating in a $30 \mathrm{~min}$ physical activity training for 4 days in a week (15). The results revealed that the peroneus longus of the non-dominant limb had a significantly shorter latency than that of the dominant limb. Based on the activity level of the subjects, the authors concluded that these results may be due to the different loads put on the non-dominant and dominant ankles during physical activity (16). Indeed, a study conducted in healthy physically active participants found higher sway velocity in the dominant leg than in the non-dominant leg during single-leg, eyes-open, or 
eyes-closed conditions (16). The authors pointed to a probable change in balance control and development of specific postural adaptations due to physical training $(36,37)$. Furthermore, it is also very well known that athletes place different loads on the non-dominant and dominant leg $(38,39)$. Stephens et al. $(40)$ reported differences in jumping height and maximum vertical ground reaction force between the two limbs in subjects with competitive volleyball experience. In addition, athletes who use their non-dominant limb to support body weight and maintain balance during a ball kick or stepping up onto a step exhibit better postural control in the non-dominant limb (16). The findings regarding the differences between the limbs in athletes support the debate between the authors that the difference in loads put on the non-dominant and dominant limbs leads to assessable differences in strength, postural balance, and muscle latency.

There could be possible links why muscle reaction time would be different between the limbs in individuals with physical training experience. The different activity related loads put on the non-dominant and dominant limb may cause an alteration in muscle spindle activity. Static and dynamic gamma motor neurons send stimuli to the muscle spindles, which act as stretch receptors. Gamma motor neurons can adjust the sensitivity of the intrafusal fibers by innervating these intrafusal fibers of the muscle spindle (41). Several investigators already showed increased muscle activity following joint afferent fiber or mechanoreceptor stimulation and activation of gamma motor neurons $(42,43)$. An increase in firing of the motor nerves to the muscles due to the activations of the muscle spindles following stretching that may potentially boost the concentric force of contraction in the muscle fibers was also expressed by Dietz et al. (44). Therefore, it is considered that the unanticipated stretch caused by a supination perturbation changes the dynamic gamma motor neuron activity and could make the muscle spindles of the peroneus or tibial muscles more or less sensitive. As a result, it can be hypothesized that the alteration in the sensitivity of the gamma motor neuron activity of the dominant limb due to different activity related demands placed on the dominant leg compared with the non-dominant leg may lead to a decrease in the recruitment of motor nerves to the muscles and cause a decreased reflexive activation in the dominant limb. The fact that healthy sedentary individuals did not put different loads on the non-dominant and dominant limbs explains why we could not find differences in muscle reaction time between the limbs. Yeung et al. (20) and Ekstrand et al. (21) reported in their studies that the dominant ankle was sprained 2-2.4 times more frequently than the non-dominant ankle in athletes. Knight et al. $(15,16)$ concluded in their studies that the poorer balance or latency of the peroneus longus muscle of the dominant limb may help explain why the dominant ankle is sprained more frequently than the non-dominant ankle. Based on our results, it can also be hypothesized that in healthy sedentary individuals there is no difference in the risk of ankle sprain between the limbs. However, further studies that assess ankle injuries with a similar subject group are necessary.

There are few studies that have investigated differences in proprioceptive ability (joint position sense or kinesthesia) between the non-dominant and dominant legs of healthy sedentary individuals $(45,46)$. Fischer-Rasmussen et al. $(45)$ performed angle reproduction and detection of passive movement tests of the knee joints in healthy sedentary individuals. They could not present significant differences between the non-dominant and dominant limbs with respect to the ability to reproduce an angle to a certain position and detect passive movement. Aydın et al. (46) also observed no significant differences between nondominant and dominant ankles with respect to the ability to sense passive movement and the active angle-reproduction test results in sedentary subjects, who were included in the study as a control group. Our results correlate with the results of these studies, with no differences found between the limbs with respect to joint position sense and kinesthesia $(45,46)$ in active individuals [teenage female gymnasts (46), a mixed group of male and female elite athletes $(22,23)$, and female handball players (47)]. Interestingly, there were also no differences between the ankle joints with respect to joint position sense.

\section{Conclusion}

The results of this investigation indicate that dominance does not interfere with the evaluation of reaction times of peroneus longus or tibialis anterior muscles, ankle joint position sense, or ankle kinesthesia in healthy sedentary individuals. Clinicians and researchers may find these results of particular importance when performing comparative evaluations between the non-dominant and dominant limbs for the purposes of either tracking changes following exercise interventions or identifying deficits in injured individuals. Our results indicate that either the dominant or non-dominant limb can be used as a reference. Further studies are needed to reach an accurate conclusion regarding ankle injuries.

Ethics Committee Approval: Ethics committee approval was received for this study from the ethics committee of Uludağ University, Faculty of Medicine (25.01.2011/ File Number: 2011-3/13).

Informed Consent: Written informed consent was obtained from participants who participated in this study.

Author contributions: Concept - U.Ş., H.G.; Design - U.Ş., H.G.; Supervision - U.Ş., H.G.; Resource - U.Ş., S.B.K.; Materials - S.B.K., H.G.; Data Collection and/or Processing - U.Ş., S.B.K.; Analysis and/or Interpretation - U.Ş., S.B.K., H.G.; Literature Search - U.Ş., S.B.K.; Writing - U.Ş., S.B.K.; Critical Reviews - H.G.

Conflict of Interest: No conflict of interest was declared by the authors.

Financial Disclosure: The authors declared that this study has received no financial support. 


\section{References}

1. Konradsen L, Beynnon BD, Renstrom PA. Proprioception and sensorimotor control in the functionally unstable ankle. In: Lephart SM, $\mathrm{Fu} \mathrm{FH}$, editors. Proprioception and neuromuscular control in joint stability 1 st ed. Champaign, IL: Human Kinetics; 2000. p. 237-46.

2. Docherty $\mathrm{CL}$, Arnold $\mathrm{BL}$. Force sense deficits in functionally unstable ankles. J Orthop Res 2008;26:1489-93. [CrossRef]

3. Forkin DM, Koczur C, Battle R, Newton RA. Evaluation of kinesthetic deficits indicative of balance control in gymnasts with unilateral chronic ankle sprains. J Orthop Sports Phys Ther 1996;23:245-50. [CrossRef]

4. Hertel J, Olmsted-Kramer LC. Deficits in time-toboundary measures of postural control with chronic ankle instability. Gait Posture 2007;25:33-9. [CrossRef]

5. McKeon PO, Hertel J. Spatiotemporal postural control deficits are present in those with chronic ankle instability. BMC Musculoskelet Disord 2008;9:76. [CrossRef]

6. Mitchell A, Dyson R, Hale T, Abraham C. Biomechanics of ankle instability. Part 2: postural sway-reaction time relationship. Med Sci Sports Exerc 2008;40:1522-8. [CrossRef]

7. Hartsell HD, Spaulding SJ. Eccentric/concentric ratios at selected velocities for the invertor and evertor muscles of the chronically unstable ankle. Br J Sports Med 1999;33:255-8. [CrossRef]

8. Sekir U, Yildiz Y, Hazneci B, Ors F, Aydin T. Effect of isokinetic training on strength, functionality and proprioception in athletes with functional ankle instability. Knee Surg Sports Traumatol Arthrosc 2007;15:654-64. [CrossRef]

9. Konradsen L, Ravn JB. Prolonged peroneal reaction time in ankle instability. Int J Sports Med 1991;12:290-2. [CrossRef]

10. Lofvenberg R, Karrholm J, Sundelin G, Ahlgren O. Prolonged reaction time in patients with chronic lateral instability of the ankle. Am J Sports Med 1995;23:414-7. [CrossRef]

11. Mitchell A, Dyson R, Hale T, Abraham C. Biomechanics of ankle instability. Part 1: reaction time to simulated ankle sprain. Med Sci Sports Exerc 2008;40:1515-21. [CrossRef]

12. Ozdemir RA, Pourmoghaddam A, Paloski WH. Sensorimotor posture control in the blind: Superior ankle proprioceptive acuity does not compensate for vision loss. Gait Posture 2013;38:603-8. [CrossRef]

13. Han KM, Ricard MD. Effects of 4 weeks of elastic-resistance training on ankle-evertor strength and latency. J Sport Rehabil 2011;20:157-73.

14. Henry B, McLoda T, Docherty CL, Schrader J. The effect of plyometric training on peroneal latency. J Sport Rehabil 2010;19:288300.

15. Knight AC, Weimar WH. Difference in response latency of the peroneus longus between the dominant and nondominant legs. J Sport Rehabil 2011;20:321-32.

16. Knight AC, Weimar WH. Effects of ankle taping on single and double leg balance. Sport Science Review 2010;19:1-19. [CrossRef]

17. Lina WH, Liub YF, Hsieh CC, Lee AJ. Ankle eversion to inversion strength ratio and static balance control in the dominant and non-dominant limbs of young adults. J Sci Med Sport 2009;12:42-9.[CrossRef]

18. Alonso AC, Brech GC, Bourquin AM, Greve JM. The influence of lower-limb dominance on postural balance. Sao Paulo Med J 2011;129:410-3.

19. Hoffman M, Schrader J, Applegate T, Koceja D. Unilateral postural control of the functionally dominant and nondominant extremities of healthy subjects. J Athl Train 1998;33:319-22.
20. Yeung MS, Chan KM, So $\mathrm{CH}$, Yuan WY. An epidemiological survey on ankle sprains. Br J Sports Med 1994;28:112-6. [CrossRef]

21. Ekstrand J, Gillquist J. Soccer injuries and their mechanisms: a prospective study. Med Sci Sports Exerc 1983;15:267-70. [CrossRef]

22. Halasi T, Kynsburg A, Tallay A, Berkes I. Changes in joint position sense after surgically treated chronic lateral ankle instability. $\mathrm{Br} J$ Sports Med 2005;39:818-24. [CrossRef]

23. Kynsburg A, Halasi T, Tallay A, Berkes I. Changes in joint position sense after conservatively treated chronic lateral ankle instability. Knee Surg Sports Traumatol Arthrosc 2006;14:1299-306. [CrossRef]

24. Keles SB, Sekir U, Gur H, Akova B. Eccentric/concentric training of ankle evertor and dorsiflexors in recreational athletes: muscle latency and strength. Scand J Med Sci Sports 2014;24:29-38. [CrossRef]

25. Keles SB, Sekir U, Akova B, Gur H. Test-retest reliability of peroneal reaction time using a custom designed inversion tilting platform in healthy recreational athletes. In: Korkusuz F, Ertan H, Tsolakidis E, editors. Proceedings of the 15th Annual Congress of the European College of Sport Science; 2010 June 23-26; Antalya, Turkey. p. 342.

26. Currier DP. Elements of research in physical therapy, 3rd ed. Baltimore, MD: Williams and Wilkins; 1990.

27. Sekir U, Yildiz Y, Haneci B, Ors F, Saka T, Aydin T. Reliability of a functional test battery evaluating functionally, proprioception, and strength in recreational athletes with functional ankle instability. Eur J Phys Rehabil Med 2008;44:407-15.

28. Eechaute C, Vaes P, Duquet W, Gheluwe BV. Test-retest reliability of sudden ankle inversion measurements in subjects with healthy ankle joints. J Athl Training 2007;42:60-5.

29. Lynch SA, Eklund U, Gottlieb D, Renstrom PA, Beynnon B. Electromyographic latency changes in the ankle musculature during inversion moments. Am J Sports Med 1996;24:362-9. [CrossRef]

30. Hopkins JT, Brown TN, Christensen L, Palmieri-Smith RM. Deficits in peroneal latency and electromechanical delay in patients with functional ankle instability. J Orthop Res 2009;27:1541-46. [CrossRef]

31. Henry B, McLoda T, Docherty CL, Schrader J. The effect of plyometric training on peroneal latency. J Sport Rehab 2010;19:288-300.

32. Konradsen $L$, Voigt $M$, Hajjsgaard $C$. Ankle inversion injuries: the role of the dynamic defense mechanism. Am J Sports Med 1997;25:548. [CrossRef]

33. Eils E, Rosenbaum D. A multi-station proprioception exercise program in patients with ankle instability. Med Sci Sports Exerc 2001;33:1991-8. [CrossRef]

34. Hopkins TJ, McLoda T, McCaw S. Muscle activation following sudden ankle inversion during standing and walking. Eur J Appl Physiol 2007;99:371-8. [CrossRef]

35. Mitchell A, Dyson R, Hale T, Abraham C. Biomechanics of ankle instability. Part 1: reaction time to simulated ankle sprain. Med Sci Sports Exerc 2008;40:1515-21. [CrossRef]

36. Hugel F, Cadopi M, Kohler F, Perrin P. Postural control of ballet dancers: a specific use of visual input for artistic purposes. Int J Sports Med 1999;20:86-92. [CrossRef]

37. Perrin PP, Bene MC, Perrin CA, Durupt D. Ankle trauma significantly impairs posture control: a study in basketball players and controls. Int J Sports Med 1997;18:387-92. [CrossRef]

38. Carey DP, Smith G, Smith DT, Shepherd JW, Skriver J, Ord L, et al. Footedness in world soccer: an analysis of France '98. J Sports Sci 2001;19:855-64. [CrossRef] 
39. Jacobs C, Uhl TL, Seeley M, Sterling W, Goodrich L. Strength and fatigability of the dominant and nondominant hip abductors. J Athl Train 2005;40:203-6.

40. Stephens TM, Lawson BR, DeVoe DE, Reiser RF. Gender and bilateral differences in single-leg countermovement jump performance with comparison to a double-leg jump. J Appl Biomech 2007;23:190-202.

41. Kandel ER, Schwartz JH, Jessell TM. Spinal reflexes. In: Butler J, Leowitz $\mathrm{H}$, editors. Principles of Neural Science. 4th ed. New York, NY: McGraw-Hill; 2000. p. 713-36.

42. Freeman MA, Wyke B. Articular reflexes at the ankle joint: an electromyographic study of normal and abnormal influences of ankle joint mechanoreceptors upon reflex activity in the leg muscles. $\mathrm{Br}$ Surg 1967;54:990-1001. [CrossRef]
43. Johansson $\mathrm{H}$, Sjolander P, Sojka P. Actions on gamma-motoneurones elicited by electrical stimulation of joint afferent fibres in the hind limb of the cat. J Physiol 1986;375:137-52. [CrossRef]

44. Dietz V, Schmidtbleicher D, Noth J. Neuronal mechanisms of human locomotion. J Neurophysiol 1979;42:1212-22.

45. Fischer-Rasmussen T, Jensen PE. Proprioceptive sensitivity and performance in anterior cruciate ligament-deficient knee joints. Scand J Med Sci Sports 2000;10:85-9. [CrossRef]

46. Aydin T, Yildiz Y, Yildiz C, Atesalp S, Kalyon TA. Ankle proprioception: a comparison between female teenage gymnasts and controls. Physical Medicine 2000;3:11-20.

47. Panics G, Tallay A, Pavlik A, Berkes I. Effect of proprioception training on knee joint position sense in female team handball players. $\mathrm{Br}$ J Sports Med 2008;42:472-6. [CrossRef] 